Mirai. Estudios Japoneses

ISSN-e: 1988-2378

http://dx.doi.org/10.5209/MIRA.57405

\title{
Coleccionando arte contemporáneo Japonés en España: El caso de Alfonso González-Calero
}

\author{
Alejandra Rodríguez Cunchillos ${ }^{1}$
}

Resumen: La presente comunicación tiene como objeto analizar la evolución de la colección de arte contemporáneo japonés del coleccionista madrileño Alfonso González-Calero (Madrid, 1958), a partir de determinadas circunstancias vitales que han permitido su conformación. Ingeniero en tasación y valoración de obras, crítico, comisario y galerista, comenzó su andadura en el mundo del arte a edad muy temprana, organizando su primera exposición con tan solo diecinueve años. Adquirió su primera obra en la década de los ochenta, coincidiendo con la creación de nuevos espacios de exhibición, museos de arte contemporáneo, revistas especializadas o ferias de arte, que permitieron dar a conocer al coleccionista español las nuevas propuestas artísticas nacionales e internacionales y, entre ellas niponas. Asimismo, aumentó el número de artistas foráneos que elegían como destino nuestro país, como es el caso de la artista Totoki Michiko, junto con quien Alfonso González-Calero se sumergió en la cultura japonesa. Posteriormente y de manera fortuita, conoció al comisario de arte japonés Minematsu Hideki con quien crearía el grupo de artistas japoneses Non Stop, formado por artistas de muy diversas disciplinas, y con el objetivo de sobrepasar el arte tradicional y enseñar su obra en el extranjero, y por ende en España.

De esta manera, en la actualidad Alfonso González-Calero posee una amplia colección de arte contemporáneo japonés, siendo uno de los coleccionistas más destacados en esta línea en nuestro país. Palabras clave: Arte contemporáneo japonés; coleccionismo español; coleccionistas; Non Stop.

Cómo citar: Rodríguez Cunchillos, A. (2017). Coleccionando arte contemporáneo Japonés en España: El caso de Alfonso González-Calero (2017), en Mirai. Estudios Japoneses 1, 2017, 317-331.

\section{[es] Collecting Japanese contemporary art in Spain: The case of Alfonso González-Calero}

\begin{abstract}
This essay aims to analyze the development of the Japanese contemporary art collection owned by Alfonso González-Calero (Madrid, 1958), taking as a starting point certain life circumstances which have led to its formation. González-Calero is a Project Appraisal and Evaluation Engineer, art critic, curator and gallerist. His career in the world of art commenced at an early age, curating his first exhibition at the age of nineteen. In addition, his interest in art grew during the eighties, when he acquired his first work of art. At that time, there were new exhibition spaces, museums of contemporary art as well as the first international contemporary art fair in Spain (ARCO), which allowed Spanish collectors to learn about the new national and international trends in the art world, and among them Japanese proposals. As a result, the number of foreign artists who chose our country as a destination increased, and among them there were Japanese artists such as Totoki Michiko, who helped Alfonso González-Calero to immerse himself in Japanese culture. Later, by chance, he met the curator of Japanese art Minematsu Hideki, with whom he created the group Non Stop, formed by Japanese artists from many different disciplines, that aimed to surpass traditional art and show their work abroad, and thus in Spain.
\end{abstract}

Universidad de Zaragoza.
a.rguez.cunch@gmail.com 
As a consequence of all these circumstances, Alfonso González-Calero has an extensive collection of Japanese contemporary art, being one of the most important collectors of this type of art in Spain.

Keywords: Contemporary Japanese art; Spanish collectors; collecting; Non Stop.

"Me ha preocupado menos el valor económico o el prestigio y me he guiado más por el valor estético o la calidad"

Alfonso González-Calero²

Alfonso González-Calero y González (Madrid, 1958)³, nació en un entorno familiar que contaba con ciertos medios económicos, a pesar de los estragos que la Guerra Civil había ocasionado. Cabe recordar que por estas fechas el acceso a la cultura era limitado, aun así su familia había conservado una colección de libros, la cual le permitió al coleccionista acercarse a la literatura y al arte, como catálogos de grandes maestros de las bellas artes de la editorial Rizzoli ${ }^{4}$, que coleccionaba su padre. Podríamos decir, que éstos fueron una de sus primeras conexiones con el arte. Asimismo, el hecho de vivir en el barrio madrileño de las Letras, concretamente en la Calle Huertas, a escasos metros de Museo del Prado le permitió conocer éste de primera mano. Para el coleccionista, durante su niñez este museo no solamente fue un lugar para jugar y corretear, tanto en los exteriores como en los interiores del mismo - hay que tener en cuenta que por estas fechas las restricciones y medidas de seguridad del museo no tenían parangón con las actuales - sino que con el tiempo se convirtió en un espacio en el que dar rienda suelta a su imaginación y visitar a menudo para contemplar sus obras. . Podríamos decir, que éstos fueron los puntos de partida de Alfonso González-Calero para adentrarse en mundo del arte.

En cuanto a su interés por el coleccionismo, apareció durante su infancia. El hecho de agrupar diferentes objetos como canicas, cromos o coleccionables de la época, y poseer varios de un tipo en concreto, así como de reunir elementos que le permitieran diferenciarse del resto, era un aspecto que entusiasmaba al coleccionista y que ha continuado hasta nuestros días.

2 Quiero agradecer y resaltar la implicación que Alfonso González-Calero y González ha tenido con esta investigación, proporcionándonos todos los datos que le hemos solicitado y mostrando una intachable disponibilidad. Igualmente, nos ha aportado valiosos documentos como artículos, entrevistas inéditas o documentos de su archivo privado, imprescindibles para elaborar este estudio.

3 Respecto al coleccionista y su colección véanse las siguientes entrevistas: SALAs, Ana (2010): “Con 15 años me quedé una noche encerrado en el Museo del Prado". En El comercio.es, Madrid, 7 de junio de 2010, http:// www.elcomercio.es/v/20100607/oviedo/anos-quede-encerrado-noche-20100607.html [19/02/16]; PoDRAN, Gianna (2016); “Cinco preguntas a Alfonso González Calero". En AECASpain, Madrid, 15 de febrero de 2016, http:/www.aecaspain.es/index.php/noticias/encuentros-con/425-cinco-preguntas-a-alfonso-gonzalez-calero [19/02/16]. Asimismo, las entrevistas que realizamos en el espacio expositivo Art Room, en Madrid en abril de 2015.

$4 \quad$ Rizzoli es una editorial fundada por Angelo Rizzoli en 1909 en Italia. Entre muchas de sus publicaciones estaban también las de catálogos de arte.

5 Alfonso González Calero cuenta en una entrevista cómo se divertía en el Museo del Prado: “Con 15 años me quedé una noche encerrado en el museo. Yo jugaba con mi hermano allí, una noche cerraron y nos quedamos dentro. Estuvimos a oscuras por las salas un buen rato... Cuando el conserje nos vio se dio un buen susto [...]He vivido en la calle Huertas y siempre íbamos a jugar al Prado. Jugábamos a las chapas, íbamos por los sótanos, los tejados... Hoy no te dejarían. Pero aquel era un casón enorme que lo tomábamos como nuestra segunda casa” en SAlas, Ana (2010): “Con 15 años...op. cit. 


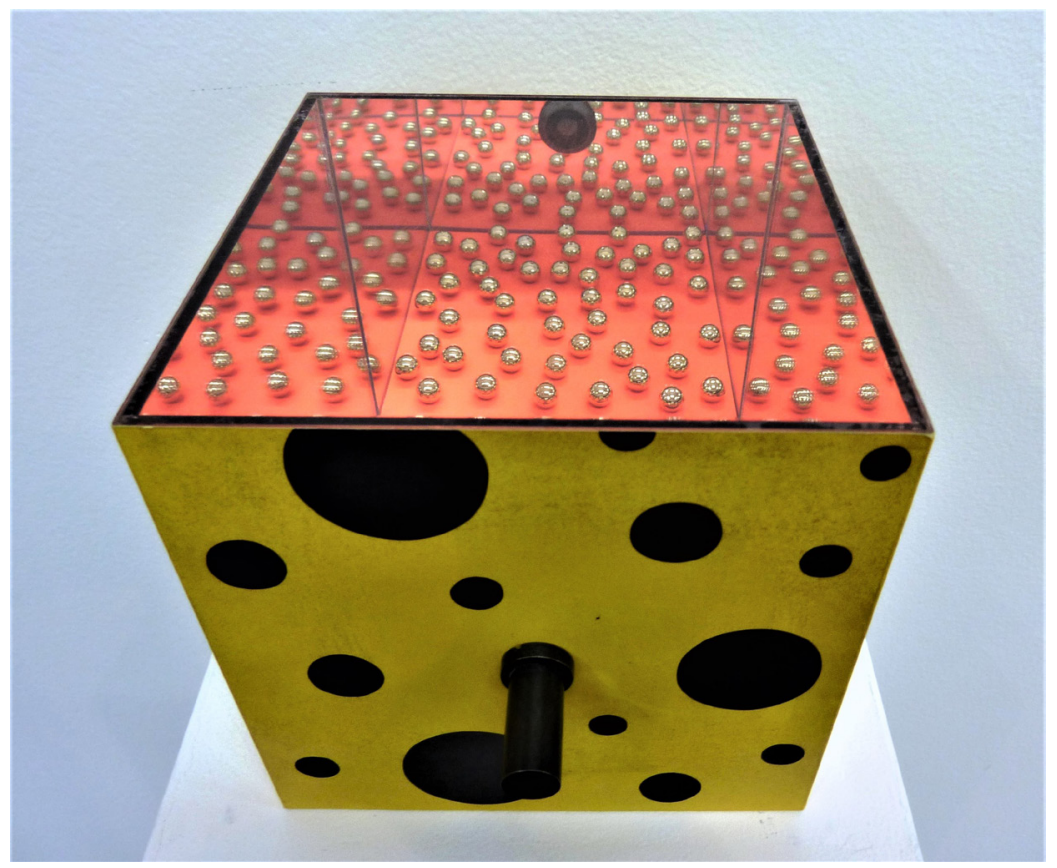

Fig. 1. Kusama Yayoi, Mirror Box, 1992, serigrafía, plexiglás con espejo y bolas, 14 x14 x 14 cm., Colección particular Alfonso González-Calero y González.

De esta manera, su interés tanto por el arte como por la compilación de objetos, le llevó a montar su primera exposición con tan solo diecinueve años ${ }^{6}$, y tiempo después, a mediados de los ochenta a adquirir su primera obra: una versión de Atalanta e Hipómenes de Guido Reni (1618-19, Museo del Prado, Madrid), de la autoría de Adolfo Falcés (Madrid, 1964) ${ }^{7}$, un amigo pintor. Alfonso González-Calero nos contaba cómo fue este momento en una entrevista: "es un cuadro que a mí siempre me fascinó. Este amigo pintor, hizo una versión y como sabía que la de Guido Reni no podría tenerla nunca, pensé que podría tener en mi casa esta versión que me recuerda mucho a la otra...era abstracta, pero si tenía una composición y el mismo título, no era muy caro y podía pagarlo y a él le iba a venir genial"". Asimismo, el coleccionar obras de su contemporaneidad le parecía más coherente. Posteriormente, el coleccionista decidió ampliar sus conocimientos en materia artística, concretamente sobre

6 Alfonso González-Calero hizo sus pinitos como comisario de exposiciones disponiendo una pequeña muestra en el portal de su casa. Las obras correspondían a artistas conocidos suyos, y su objetivo era darles visibilidad y colaborar en la venta de su obra. Información facilitada por Alfonso González-Calero, entrevista realizada en el espacio expositivo Art Room, en Madrid en abril de 2015.

7 Adolfo Falcés es un artista madrileño, que realizó sus estudios en la Escuela Superior de Bellas Artes de San Fernando en Madrid. Ha participado en numerosas exposiciones en España y en el extranjero, y está presente en galerías y colecciones nacionales e internacionales. http:/www.arteculturapatrimonio.es/adolfo-falces [Consulta: $01 / 03 / 17]$.

8 Información facilitada por Alfonso González-Calero, entrevista realizada en el espacio expositivo Art Room, en Madrid en abril de 2015. 
procedimientos pictóricos: a través de su relación y formación con Francisco Soto Mesa ${ }^{9}$ entre 1985-1987 y cursando un Master en Apreciación del Arte Contemporáneo en el Círculo de Bellas Artes de Madrid en 1988, impartido por Julián Gallego ${ }^{10}$.

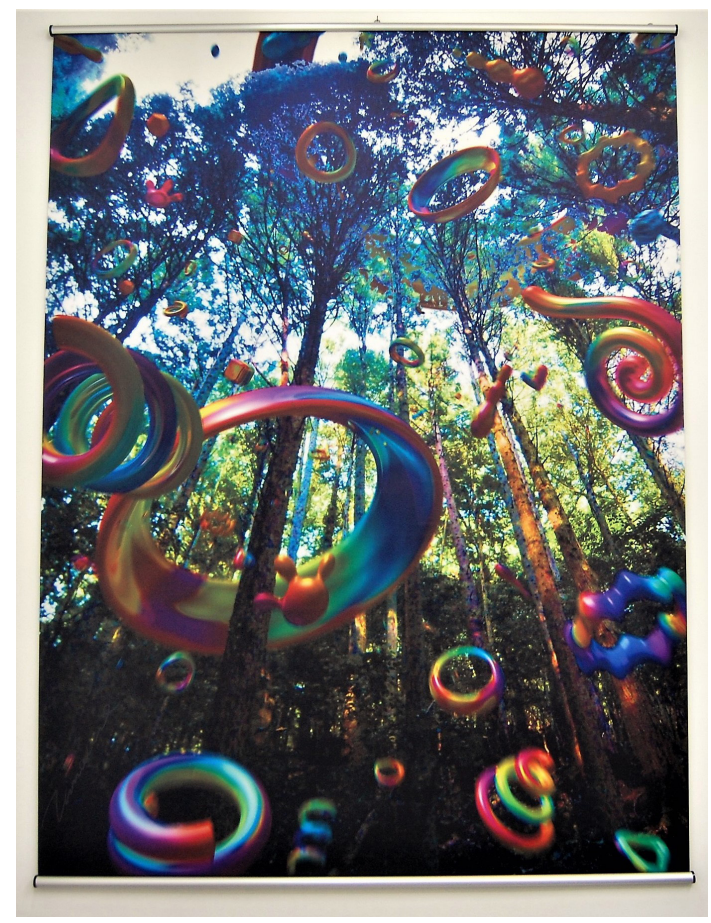

Fig. 2. Tanacue, Colorhythm on, 2009, impresión sobre tela, 150 x 200 cm., Colección particular Alfonso González-Calero y González.

Hasta el momento, hemos explicado cómo Alfonso González-Calero fue entrando contacto con el arte contemporáneo y el coleccionismo, sin embargo, nos interesa saber cómo lo hizo en este sentido con lo japonés. Fue a partir de 1986, con la llegada de la artista japonesa Tokoki Michiko ${ }^{11}$ a la capital española. Debemos decir,

$9 \quad$ Francisco Soto Mesa (Madrid, 1946) es Licenciado en Bellas Artes por la Universidad Complutense de Madrid. Desde 1978 es docente en la escuela de Artes Plásticas 'El Estudio' y organizador de exposiciones del espacio 'Sala Previa' para artistas emergentes. En 2001 inauguró junto a la escuela 'Tercer Espacio' una galería de arte centrada en exposiciones y otros eventos de artistas de prestigio, que entre 2006 y 2011 ha dirigido Rosi Rubio Liniers. http://www.franciscosotomesa.com [Consulta: 03/06/16]

10 Julián Gallego (Zaragoza, 1919 - Madrid, 2006) fue un académico, historiador y crítico de arte de pintura española de gran reconocido prestigio. Se doctoró en Historia del Arte en la Universidad de la Sorbona en París. Posteriormente ejerció como profesor en la Universidad Complutense de Madrid. Entre sus distinciones cabe destacar su admisión como académico de mérito en la Real Academia de Bellas Artes de San Fernando.

11 Totoki Michiko (1963, Tokyo) se inició en el arte desde muy pequeña; su abuela paterna tenía un estudio de pintura nihonga en Japón, fue a través de ella como entró en contacto con el arte, sobre todo con la pintura y la caligrafía. Sin embargo, la artista apostó desde muy joven por los lenguajes contemporáneos, muestra de ello son las clases tomadas de danza contemporánea o piano electrónico. Se licenció en Bellas Artes por la Universidad de Musashino en Tokyo en 1986 y se doctoró en la Universidad Complutense de Madrid con una Tesis 
que no era el primer viaje que Totoki Michiko realizaba a nuestro país, puesto que en 1982 había visitado Madrid durante ocho días por recomendación paterna ${ }^{12}$. España, para muchos artistas extranjeros era y sigue siendo un país de referencia artística. Lo cierto es que este primer viaje marcó de tal manera a la artista que en 1986 se instaló en nuestro país para llevar a cabo sus estudios de doctorado en la Universidad Complutense de Madrid. Por estas fechas, para Totoki Michiko la capital española era un lugar rico culturalmente, en palabras de la artista: "con una sociedad de espíritu bohemio y ensoñador"13, que chocaba drásticamente con su país natal.

Es en este contexto, donde conoce al coleccionista y comienzan a llevar a cabo proyectos juntos. Aunque Alfonso-González Calero tenía un ligero conocimiento sobre el país del sol naciente, será a partir de este momento cuando se impregne de él, especialmente a partir de 1992 cuando realizó su primer viaje a Japón junto a la artista, con un fin concreto: "la primera vez que viajé a Japón fue para conocer a los que iban a ser mis suegros, a pedir la mano de Michiko" nos confiaba el coleccionista en una entrevista ${ }^{14}$. En esta primera visita, además, incorporó a su colección la primera pieza japonesa, denominada por él "la estrella de la colección": una caja-objeto de la artista japonesa Kusama Yayoi, Mirror box [fig. 1], adquirida en una pequeña galería de Tokyo. A este viaje le siguieron muchos otros, no solamente con fines turísticos, sino también siempre con algún nuevo objetivo relacionado con el arte: ferias, exposiciones, eventos artísticos...en un primer momento introducido en este nuevo panorama artístico por Totoki Michiko.

Como hemos mencionado anteriormente, Alfonso González-Calero se había iniciado en el coleccionismo de arte contemporáneo durante la década de los ochenta. Sin embargo, será a partir de los noventa cuando la adquisición de este tipo de obras se haga más continua. Un dato a destacar, es la apertura de su primera galería de arte Catarsis $^{15}$ en 1992, con el fin de exponer la obra de aquellos artistas cercanos a él (muchos amigos suyos). Con piezas que para él tenían cierto valor artístico y debían de ser mostradas al público, era el arte que le rodeaba. Alfonso González-Calero nos contaba: "a diferencia de otros galeristas, nunca he ido a buscar a un artista concreto, no me ha parecido necesario [...] Me pueden criticar que no eran muy buenas, pero era lo que tenía [...] Pensé, yo de qué le puedo servir a Tàpies o a Guinovart que son artistas que ya tienen galerías, yo no necesito a Tàpies ni él a mí, pero sí a los artistas de mi alrededor que son de mi edad"16. La actividad de galerista le llevó a adquirir piezas de manera más intensa, en un primer momento de artistas españoles, muchos de ellos expuestos en la galería Catarsis — algunas piezas se quedaban en

Doctoral sobre Estética erótica: lenguajes y significados hasta el arte actual en 1993. Información facilitada por Totoki Michiko, entrevista realizada telemáticamente en septiembre de 2016. Ha participado en numerosas exposiciones, destacando las realizadas en Japón y España, véase GonZÁlez CALERo, Alfonso (2016): Memoria de actividades Art Room 2017-2018. Madrid: Art Room, pp. 62-64.

12 El padre de Totoki Michiko era ingeniero aeronáutico. Por este motivo conocía muy bien Europa y, por ende, aquellos lugares que podían interesar a su hija, Madrid era uno de ellos. Información facilitada por Totoki Michiko, entrevista realizada telemáticamente en septiembre de 2016.

13 Ibídem.

14 Información facilitada por Alfonso González-Calero, entrevista realizada en el espacio expositivo Art Room, en Madrid en abril de 2015.

15 La Galería Catarsis se encontraba en la Calle Santa María, n ${ }^{\circ} 15$ en Madrid. Estuvo activa desde 1992 hasta 2008 .

16 Información facilitada por Alfonso González-Calero, entrevista realizada en el espacio expositivo Art Room, en Madrid en abril de 2015. 
sus fondos tras la exposición y otras eran compradas - y posteriormente de otras nacionalidades como japoneses, como hemos mencionado anteriormente, en un primer momento por recomendación de Totoki Michiko.

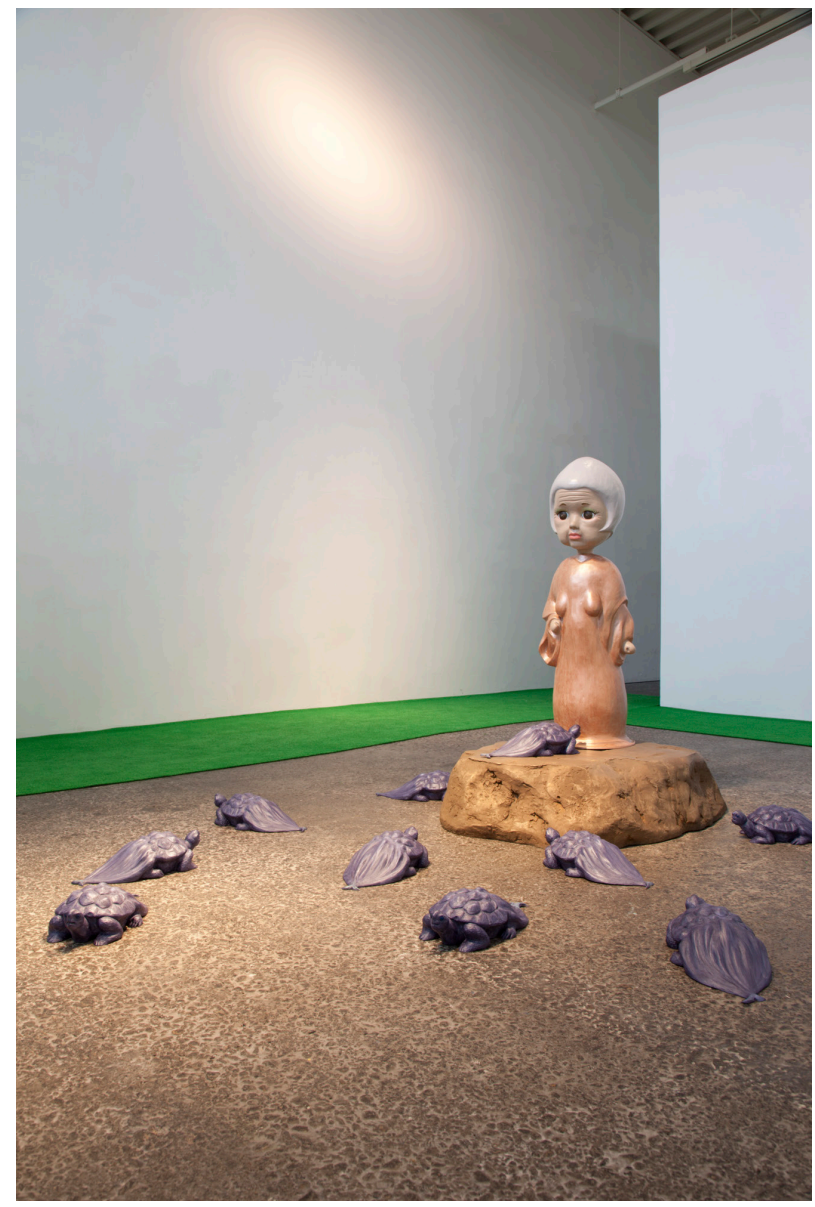

Fig. 3. Okamoto Motoko, Viewing waterfall, 2011, Modelado de resina frp, variables, Colección particular Alfonso González-Calero y González.

Continuó con su formación en el arte ${ }^{17}$, y este aprendizaje le llevó a replantearse su propia colección, la cual no seguía ninguna línea específica. Las obras habían sido adquiridas en función del interés artístico o sentimiento personal que habían

17 En 1993 realizó el Master Interior de un Museo y en 1997 el curso Galerías de Arte para el S. XXI ambos impartidos por el Ministerio de Educación y Cultura. En 2002 se tituló en Ingeniería en Valoración y Tasación de Obras de Arte por la Universidad Politécnica de Valencia. Posteriormente impartió docencia en: "Master Comunicación y Arte", Facultad de Ciencias de la Información de la Universidad Complutense de Madrid (20062008); Curso "Gestión y Dirección de Galerías de Arte" del Instituto Superior de Arte en Madrid (2007-2012) y Tasación y Valoración de obras de arte en el Instituto Superior de Arte de Madrid (2007-2012). Asimismo, cabe destacar su labor como perito judicial que viene desarrollando desde el año 2004 y como miembro de la Asociación Nacional de Peritos Judiciales y como tasador de obras de arte desde 1995. Ibídem. 
suscitado en ese preciso momento al coleccionista. Sin embargo, será a mediados de la década de los noventa, cuando la colección comience a tomar forma asignándole una línea a seguir; ésta será el formato 46 x 38 centímetros. Cualquier manifestación artística que cumpliese estas medidas podía ser incluida en la colección independientemente de la temática o estilo. Esta elección aportaba unidad a la colección, y además el resultado visual una vez expuestas era muy agradable. En su mayoría adquirió pintura y collage, aunque también fotografía, objetos, etc., haciéndose con un total de 150 piezas. Esta línea la continuó entre 1995 y 2008, fecha en la que decidió dejarla a un lado, dado el inconveniente que este formato le causaba, ya que no daba cabida a soportes como el video o la instalación, manifestaciones artísticas que Alfonso González-Calero deseaba incluir. Aunque el momento clave, en cuanto a la incorporación de arte contemporáneo japonés tanto a la galería Catarsis como a su colección, llegaría a finales de los años noventa, cuando por casualidad conoció al crítico y comisario japonés Minematsu Hideki ${ }^{18}$. El coleccionista cuenta en una entrevista cómo se conocieron: "Éste pasó por aquí, vio que tenía una exposición de una artista japonesa [...] nos conocimos y pensamos en qué podíamos trabajar juntos, él tenía contactos en Japón [...] la buena sintonía hizo todo" ${ }^{19}$. Minematsu Hideki había sido propietario de una revista de arte en Osaka, Art Gallery, y era un gran conocedor del arte y el mercado contemporáneo. Sin embargo, decidió dejarlo todo y venirse a nuestro país, con el fin de reactivarse profesionalmente. Como resultado de la conexión entre el coleccionista y el crítico japonés, dieron fruto numerosas exposiciones tanto en España como en Japón ${ }^{20}$, pero sobre todo cabe destacar la creación del grupo de artistas japoneses que denominaron Non Stop ${ }^{21}$.

18 Minematsu Hideki (Osaka, 1950 - Madrid, 2008) fue crítico y comisario de arte. Su trayectoria está ligada al arte contemporáneo y en concreto al grupo Non Stop.

19 Información facilitada por Alfonso González-Calero, entrevista realizada en el espacio expositivo Art Room, en Madrid en abril de 2015.

20 Algunas exposiciones celebradas en Japón son: Tokyo: Nuevo Arte Español en el Metropolitan Museum de Tokio en 1998; Osaka Art Show International Selection en Osaka en los años1999, 2000, 2000, 2002 y 2003.

21 Para conocer más sobre Non Stop véase: http://www.artgroupnonstop.org [14/06/16]. Su obra ha sido mostrada en numerosas exposiciones. En la Galería Catarsis, Non Stop en 2003, 2004, 2005, 2006 y Otros emergentes en 2007; Galería Anagma, Non Stop en Valencia en 2003 y Descubrir el arte japonés en 2004; Centro de Nuevas Tecnologías y Cultura del Ayuntamiento de Oviedo (La Lila), Non Stop Nueva realidad en Japón en 2006; Casa Colón Huelva, Grupo Non Stop, en 2007; Centro de Nuevas Tecnologías y Cultura del Ayuntamiento de Oviedo (La Lila), Arte Sumergido en 2008, Círculo de Bellas Artes de Valencia, Non Stop 'Avant' en 2009; Sala Pictograma, Castellón Japón Non Stop en 2011; Galería Art Room, Hoy x hoy = futuro en 2013 y Antipoda en 2015. Véanse los siguientes artículos: ANónimo (2008): "Arte sumergido en Oviedo". En Iberarte, 17 de junio de 2008, http://www.iberarte.com/index.php/artes-plasticas/pintura/2471-arte-sumergidoa-en-oviedo [16/06/2016]; MuÑIz LóPEz, José Ramón (2008): “Arte de rompe y rasga”. En El Comercio, Oviedo, 18 de junio de 2008, http://www.elcomercio.es/gijon/20080618/oviedo/arte-rompe-rasga-20080618.html [14/06/16]; Albert, Isabel (2009): “El arte japonés en el Círculo de BBAA de Valencia”. En Arte y Libertad, Valencia, n ${ }^{\circ}$ 56, 6 de julio de 2009, http://isabelalbert8.blogspot.jp/2009/07/non-stop-arte-contemporaneo-japones-en.html [14/06/16]; Anónimo (2011):Japón Non Stop". En El Mundo, Castellón, Cultura, 16 de junio de 2011, http:// www.elmundo.es/elmundo/2011/06/14/castellon/1308047535.html [14/06/16] y ANÓNIMO (2011): “'Stop' contra los prejuicios". En Las provincias, Castellón, 17 de junio de 2011, http://www.lasprovincias.es/v/20110617/ castellon/stop-contra-prejuicios-20110617.html [14/06/16]; GonZÁlez CALERo, Alfonso (2013): Memoria de actividades 2012-2013 IV Aniversario Galería Art Room. Madrid: Art Room, p. 45; GonZÁlez CALERo, Alfonso (2015): Memoria de actividades 2014-2015 VI Aniversario Galería Art Room. Madrid: Art Room, p. 43. Véase: http://www.investigacionyarte.com/ia/non-stop/ [09/06/16]. 


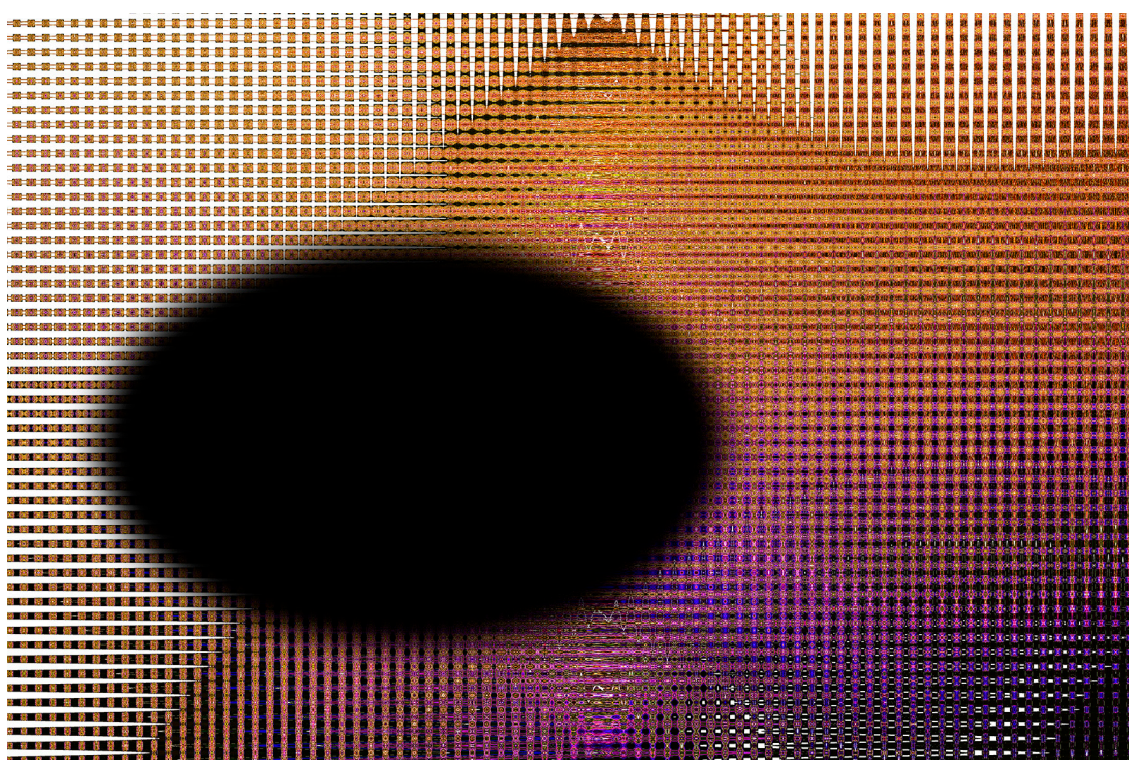

Fig. 4. Ooya Shingo, Dark energy, 2013, foto-experimento digital, variables, Colección particular Alfonso González-Calero y González.

El grupo Non Stop se fundó en 2002 —aunque previamente habían trabajado con algunos de sus integrantes - pero sería en 2005 cuando este tomaría conciencia de colectivo y mostraría su trabajo en conjunto con un propósito común: reflexionar sobre los estereotipos asociados a Japón y establecer un discurso crítico a través de sus obras ${ }^{22}$. Formado por 28 artistas nipones de disciplinas diversas: Keirin, Kinoshita Shin'ichi, Kouno Masahiro, Aoki Ruri, Sakamoto Tatsuhiko, Ogawa Takuya, Sakuma Yasuyuki, Ooya Shingo, Oonishi Satomi, Tanacue, Shimura Hidemi, Aoki Hitoshi, Takana Kouichi, Odawara Eiko, Taya Masaru, Tamura Shigenao, Nishio Shihoko, Okamoto Motoko, Mizutani Izuru, Araki Naoya, Oota Seikou, Demachi Mitsunori, Kato Hiromitsu, Kikuchi Kazuo, Nitta Shinzi, Inaba Takashi, Namura Misa y Kitamura Naoto. El crítico de arte japonés, Minematsu Hideki actuaría como puente entre Japón y España, en palabras de Alfonso González-Calero: "Hideki cumplía un papel importantísimo dentro de este proyecto, pues era el encargado de transmitir nuestras ideas a los artistas japoneses implicados en el mismo, además de ser, por su encanto y carisma el auténtico alma mater de toda esta iniciativa"23.

22 «Son sus mejores creaciones, y las que nunca podrán exponer en su propio país, allí las reglas sociales y la tradición lo impiden», explicaba Carmen Aragón, organizadora de la muestra Rompe y rasga en 2008, MuÑIZ López, José Ramón (2008): “Arte de rompe y... op. cit.

23 Folleto de la exposición Arte Sumergido de Non Stop celebrada del 18 al 30 de junio de 2008, en el Centro de Nuevas Tecnologías y Cultura del Ayuntamiento de Oviedo (La Lila), p. 1. 


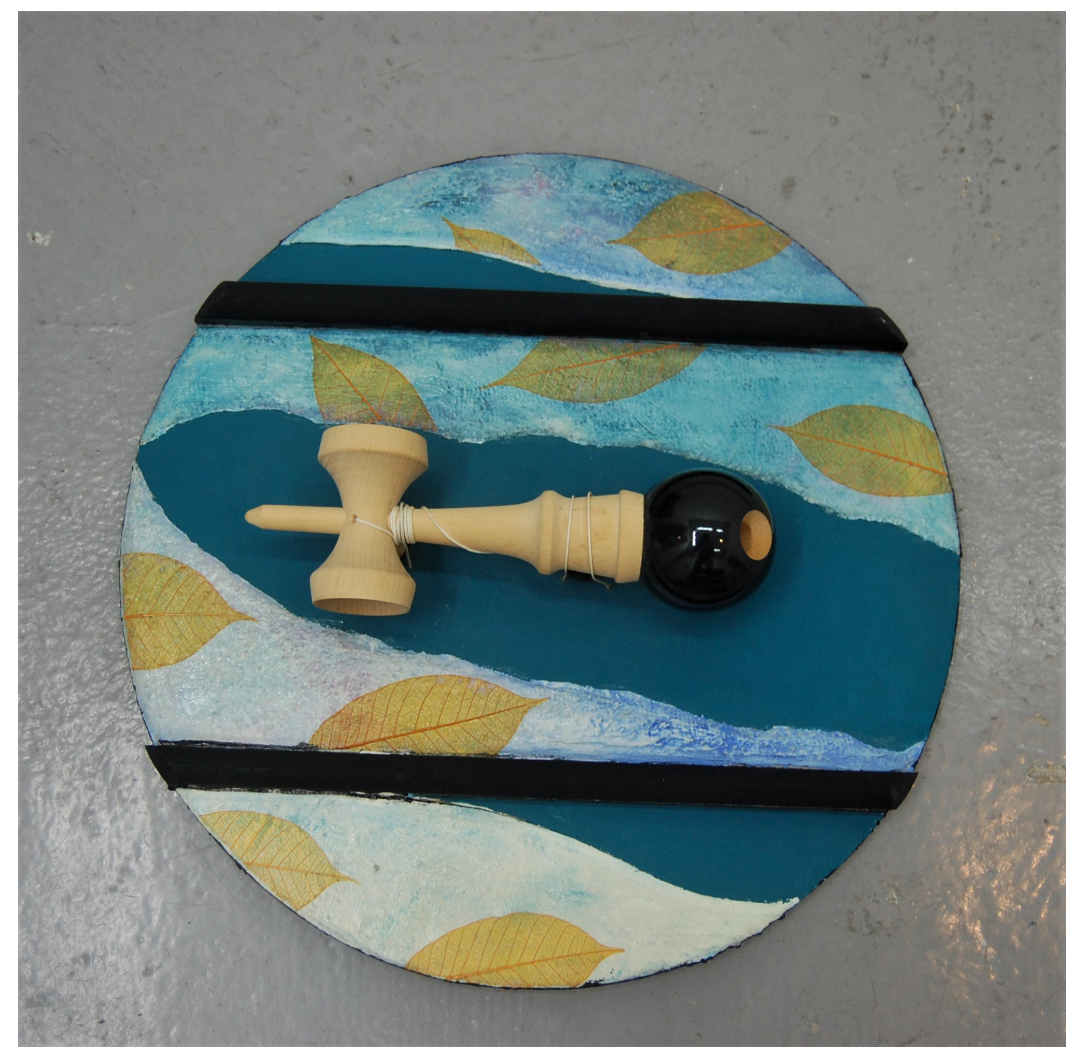

Fig. 5. Kinoshita Shin'ichi, Sin titulo, 2008, madera, objeto y técnica mixta, 36 , Colección particular Alfonso González-Calero y González.

Más allá de sobrepasar el arte tradicional, otro de los propósitos del grupo era enseñar su obra en el extranjero y por ende en España. Los artistas japoneses, incluso hoy en día, se enfrentan a un tipo de enseñanza artística jerarquizada que les resta visibilidad, y por lo tanto dificulta sus carreras artísticas. El coleccionista nos explicaba en una entrevista:

[...] el maestro crea una asociación con unos alumnos, los cuales a su vez tienen otros alumnos... Cuando el maestro hace una exposición todos los alumnos compran, o sea vende todo. Cuando la hacen los alumnos de segundo nivel venden bastante, y los del siguiente venden ya menos, solo a sus familias [...] es un sistema bastante cerrado que se alimenta no por el coleccionista ni por el degustador de arte, si no por el propio alumnado ${ }^{24}$.

24 Información facilitada por Alfonso González-Calero, entrevista realizada en el espacio expositivo Art Room, en Madrid en abril de 2015. 
Por este motivo, algunos miembros de Non Stop en desacuerdo con este modo de hacer, trataron de romper con él. Podríamos decir que, el grupo Non Stop se distinguió principalmente por este interés común. En este sentido, tanto Alfonso González-Calero como Minematsu Hideki les hicieron de trampolín para lanzarlos a un nuevo mercado, entre otros el español. Fue sobre todo el carácter rebelde y el tratarse de grupo artísticamente comprometido, recordando al histórico grupo Gutai ${ }^{25}$, lo que resultó tan interesante al coleccionista.

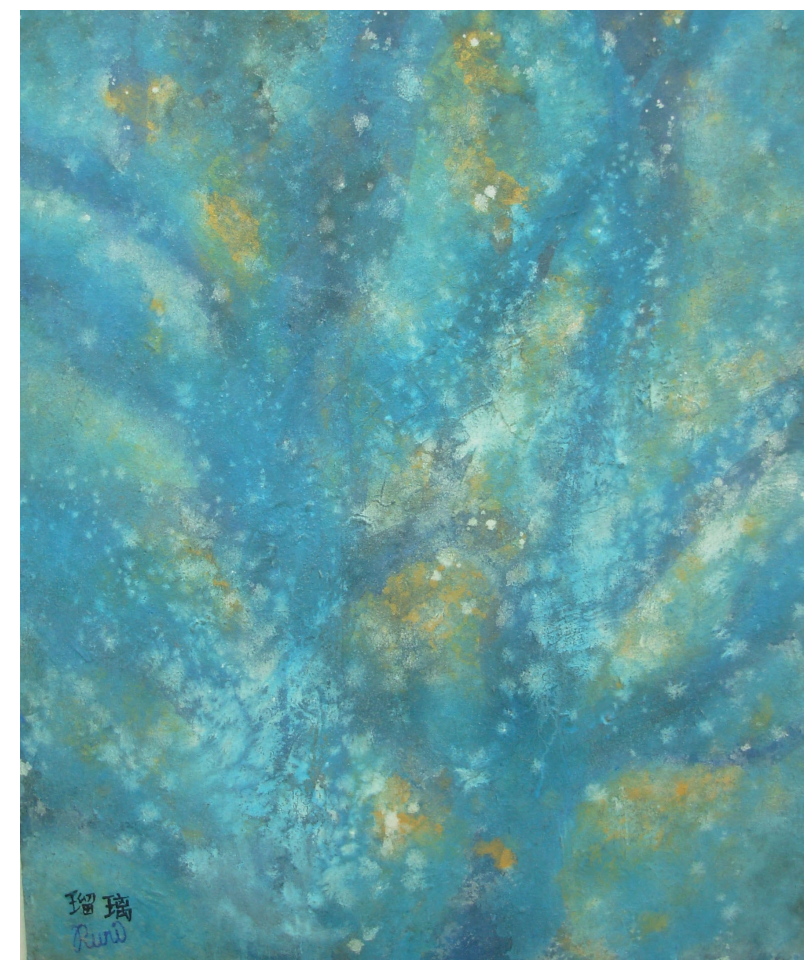

Fig. 6. Aoki Ruri, Zen, 2007, acrílico sobre lienzo, 46 x 38 cm., Colección particular Alfonso González-Calero y González.

Por esta razón, Alfonso González-Calero decidió incluirlos no solo en numerosas muestras de su galería, sino también en su propia colección ${ }^{26}$, en la que encontramos obra de artistas de estética pop como: Tanacue muy ligado al movimiento japonés 'superflat' con piezas como Colorhythm on (2009) [fig. 2] u Okamoto Motoko, quien a través de sus instalaciones reflexiona sobre el papel de la mujer o la confrontación de opuestos, como puede ser el choque cultural entre Oriente y Occidente, con la

25 El grupo Gutai (Gutai Bijutsu Kyokai) se fundó en 1954 por Yoshihara Jirō (1905-1972 Japón). En 1956 publicaron el "Manifiesto Gutai" en la revista Geijutsu Shinchō. El término Gutai procede de la suma de los kanji 具 'gu' y 体 ‘tai' y podría traducirse como 'arte concreto'. Este colectivo apostó por nuevas formas de creación y reflejó el Japón de la Postguerra. Lo llevaron a cabo a través de performances, happenings e instalaciones. A la muerte de Yoshihara Jirō en 1972 disolvieron el grupo. Véase Munroe, Alexandra (1994): Japanese Art After 1945. Scream Against The Sky. New York: Harry N. Abrams, INC., pp. 83-100.

26 Información obtenida del archivo privado de Alfonso González-Calero en abril de 2015. 
obra Viewing waterfall (2011) [fig. 3]. Por otra parte, la representación de la naturaleza es un tema muy arraigado en Japón. Así, encontramos al artista Ooya Shingo quien muestra los diferentes comportamientos de ésta, con la pieza Black hole de 2013 [fig. 4] o a Kinoshita Shin'ichi, aunque desde una estética muy diferente, con Sin título (2008) [fig. 5], en este caso recuerda a los patrones de tejidos tradicionales japoneses. Además, entre sus fondos hallamos obra de Aoki Ruri, Zen (2007) [fig. 6], artista que aboga por la belleza de la materia y las texturas en su obra, con pinceladas del zenismo. Nishio Shihoko con Fragile (2012) [fig. 7] una instalación en la que trata de jugar con la belleza de materiales comunes, entre ellos orgánicos para crear reacciones químicas naturales y ser partícipe de su transformación. Conjuntamente los fotógrafos Shinzi Nitta con The Beginning (2006) [fig. 8], quien juega con el espacio, revelando los hechos que le rodean o Demachi Mistunori con tres obras de la serie Ubu shu baga mi (2012) [fig. 9]. Estas fueron hechas tras los desastres acaecidos en Fukushima en 2011. En ellas, el artista a través de unas máscaras de cerámica trata de mostrar a los primeros humanos con deformidades provocadas por la radioactividad, un episodio que, por desgracia, azota de nuevo a Japón.

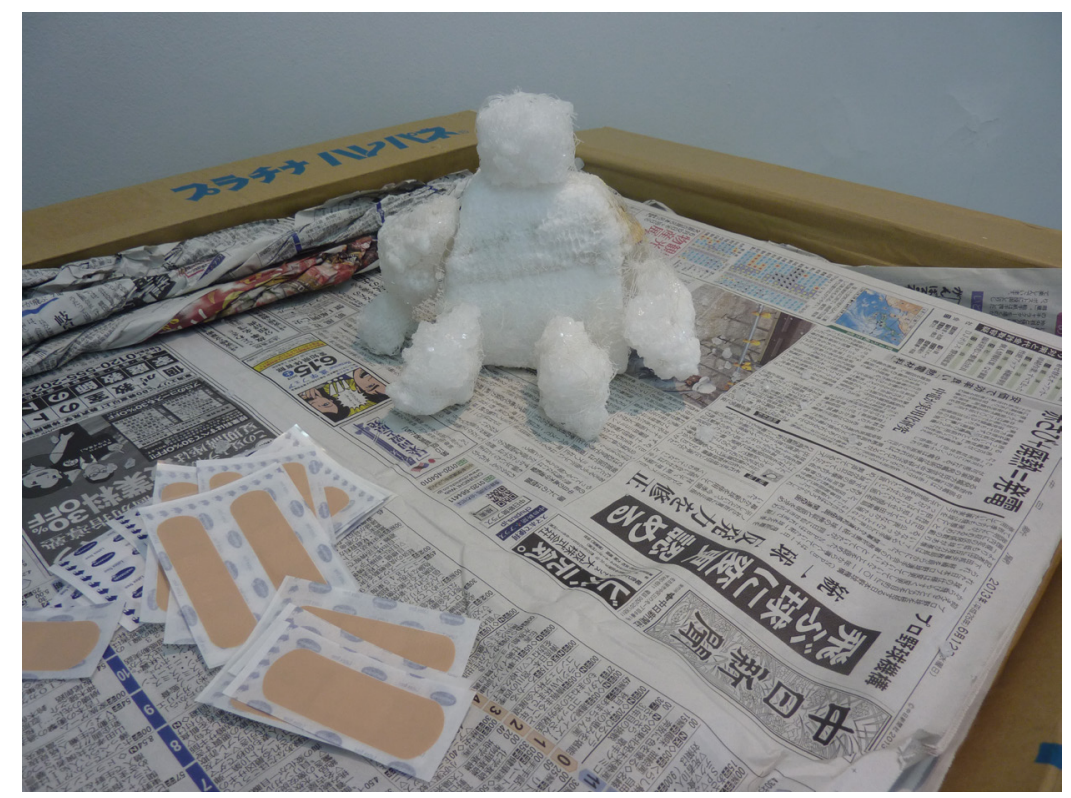

Fig. 7. Nishio Shihoko, Fragile, 2012, Caja, periódico, tiritas y muñeco de sal y red de plástico, 17 x 40 x 20 cm., Colección particular Alfonso González-Calero y González.

En 2008 fallecería su socio y amigo Minematsu Hideki y con ello se desvanecería gran parte de la esencia de este colectivo japonés. En 2010 Alfonso-González Calero decidió abrir un nuevo espacio, Investigación y Arte Sala de Arte experimental 'Art Room' en Madrid, situado en el mismo emplazamiento que la galería anterior. Desde esta fecha hasta la actualidad, ha seguido trabajando con artistas japoneses e incluso 
con integrantes del grupo Non Stop y ha dirigido otros eventos artísticos como el Festival de video arte $\mathrm{CuVo}$ en el que también se incluyen artistas nipones ${ }^{27}$.

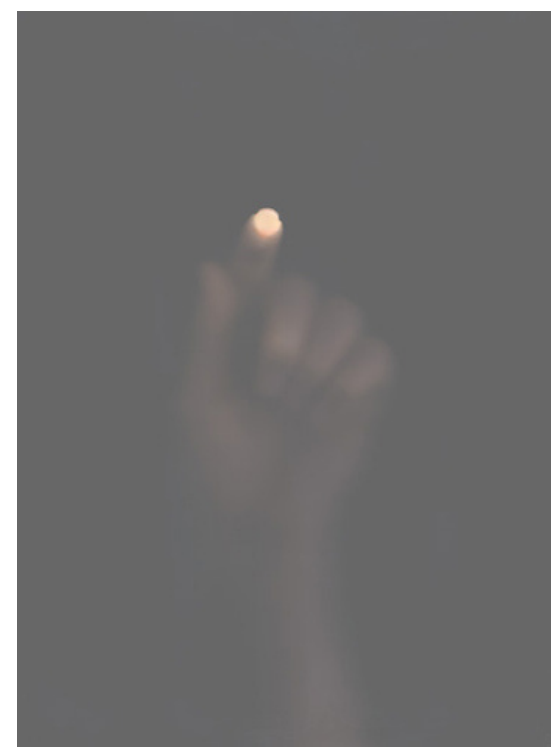

Fig. 8. Nitta Shinzi, The Beginning, 2006, fotografía digital, 51 x 72 cm., Colección particular Alfonso González-Calero y González.

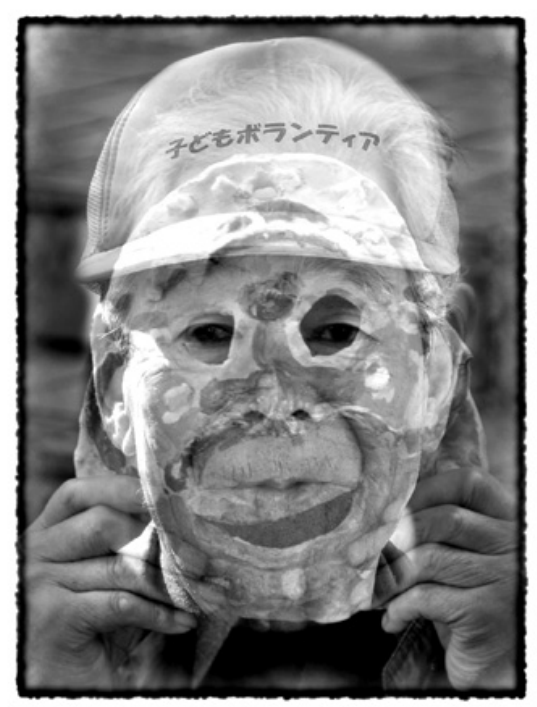

Fig. 9. Demachi Mistunori, Serie Ubu shu baga mi, 2012, fotografía digital, 30 x 20 cm., Colección particular Alfonso González-Calero y González.

27 El festival nació en 2010 bajo la denominación "Video en el cuarto" relacionado con la Feria de Arte Contemporáneo de Arévalo (Ávila). Se ha celebrado en numerosas ocasiones en otros espacios como en la Galería El Cuarto Simpático y en Art Room espacio experimental, ambos en Madrid. En él han participado artistas japoneses como: Tanacue, Totoki Michiko y Nishio Shihoko. 


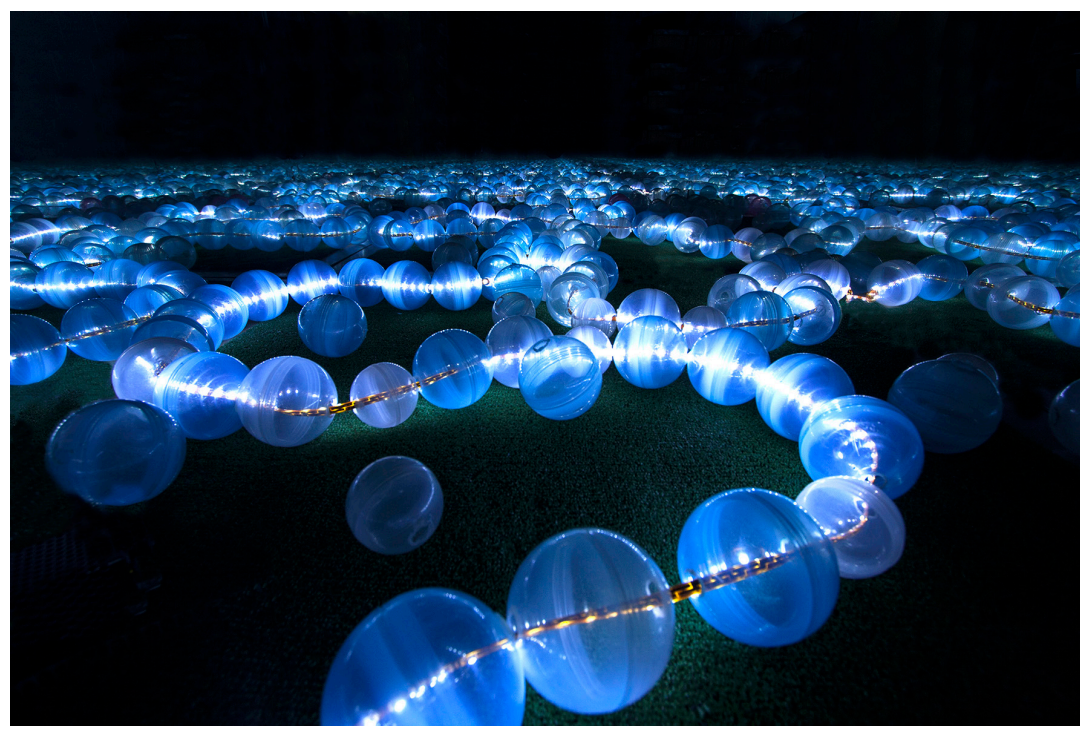

Fig. 10. Totoki Michiko, Lágrimas azules, 2001, gelatina de plata sobre papel montado en dibond., 70 x 50 cm. Colección particular Alfonso González-Calero y González.

Asimismo, a lo largo de su trayectoria ha ido integrando en su colección obra de otros artistas japoneses como, por ejemplo: Totoki Michiko, quien trabaja en diferentes disciplinas artísticas como la performance, la instalación o la fotografía, siempre tratando de evocar la energía, el ' $k i$ ', un ejemplo es la obra Lágrimas azules (2001) [fig. 10]. Morimoto Gasso, quien realiza una obra de temática histórica y religiosa, en la que presta atención a la caligrafía junto con lenguajes contemporáneos, como Katsu (2012) [fig. 11] o Katsuma Norihiko, quien superpone técnicas actuales a soportes tradicionales japoneses, por ejemplo, con Non Stop (2009) [fig. 12]. Además de otros artistas con diferentes técnicas pictóricas como: Mori Mitsuoko, Isogai Ayako, Kumon, Sanda, Yosen Onuki, Yokoshima Shoji, Sasaki Kei, Fumiko, Kyota Kyoko, Tajima Tamaki o Yamada Tomio ${ }^{28}$. En general, cabe señalar que, aunque todos ellos se mueven dentro de los parámetros del arte internacional, su obra está fuertemente enraizada con el arte y la cultura japonesa. Un aspecto sobre el arte contemporáneo japonés que el coleccionista ha resaltado:

[...] si se observa a los artistas japoneses se verá que casi siempre aparece un sustrato artístico que se nutre del tradicional ukiyo-e o de la caligrafía, al menos en lo concerniente a la supremacía de la línea, del descuido de perspectivas y de buscar la anécdota que ejemplariza, eso sí instalados desde los años 80 por un componente fuertemente pop y en algunos casos añadiendo tecnología ${ }^{29}$.

Información obtenida del archivo privado de Alfonso González-Calero en abril de 2015.

PODRAN, Gianna (2016): “Cinco preguntas a ...op. cit. 


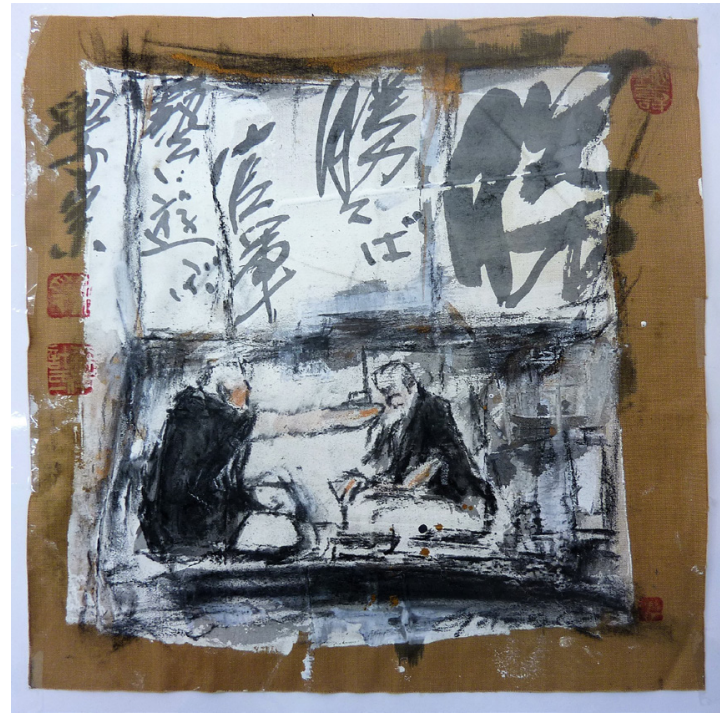

Fig. 11. Moritomo Gasso, Katsu, 2012, técnica mixta sobre obi de seda, $40 \mathrm{x}$ $40 \mathrm{~cm}$., Colección particular Alfonso González-Calero y González.

Fig. 12. Katsuma Norihiko, Non Stop, 2010, técnica mixta sobre kakejiku, 200 x $60 \mathrm{~cm}$., Colección particular Alfonso González-Calero y González.

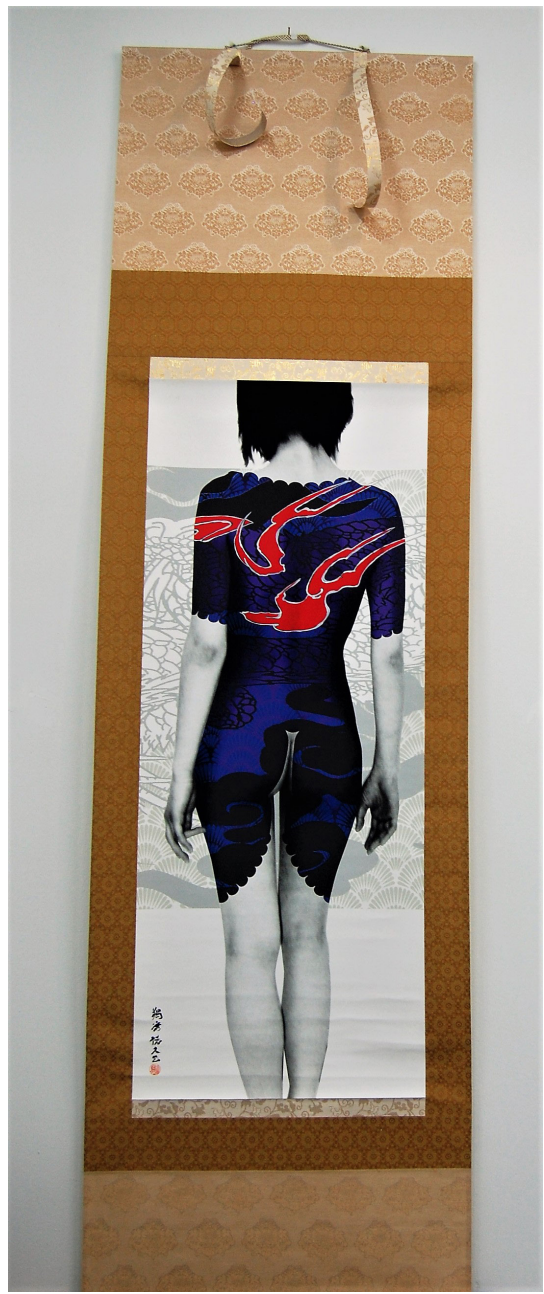




\section{Conclusiones}

A modo de conclusión, puede afirmarse que Alfonso González-Calero ha conformado una colección de gran interés para el coleccionismo de arte contemporáneo japonés en nuestro país, tanto por el elevado número de obras de arte nipón que posee, más de una cincuentena, como por la calidad de las mismas. Su colección destaca entre otras, por el hecho de haber integrado no solamente a artistas consagrados y de renombre como por ejemplo a Kusama Yayoi, si no por apostar por otros artistas con menor fama en los circuitos artísticos; como los integrantes del grupo Non Stop o artistas japoneses residentes en España, pero no por ello de menor interés, puesto que aportan personalidad a la colección y otorgan un sabor diferente al coleccionismo de arte contemporáneo japonés en España. Asimismo, su colección está compuesta por manifestaciones artísticas muy diversas tales como la pintura, la escultura, la fotografía, el video, la instalación...que van desde el zenismo, el arte experimental y conceptual, hasta las últimas tendencias artísticas como 'superflat'.

Conjuntamente, no podemos olvidar la labor llevada a cabo por Alfonso González-Calero desde su posición como galerista en nuestro país, puesto que ha promovido el conocimiento del arte contemporáneo japonés en España a través de exposiciones, edición de catálogos, ferias, etc., de la misma manera que lo ha hecho con el arte español en Japón. 